\title{
Spectroscopic Studies on Nitrogen-doped Multi-Walled Carbon Nanotubes Using Monochromated STEM-EELS at Low-Voltage
}

\author{
R. Arenal \\ Laboratorio de Microscopias Avanzadas (LMA), Instituto de Nanociencia de Aragon (INA), U. \\ Zaragoza, Zaragoza, and Fundacion ARAID, Zaragoza, Spain
}

The incorporation of nitrogen atoms into the hexagonal network of the carbon nanotube (C-NT) walls strongly modifies the chemical and physical properties of the pure C-NT [1-4]. Even if significant efforts have been devoted to investigate N-doped C-NT, considerable progresses are still needed in order to complete the understanding and optimization of their properties. In this sense, TEM is the most powerful technique for undertaking such kind of studies [1-3]. However, TEM studies on these beam-sensitive materials require working under particular conditions, low acceleration voltages and minimizing the electron dose. Thus, in this contribution we will present our studies on the local dielectric/optoelectronic properties of N-doped C-NT which will be probed by low-loss EELS developed at $80 \mathrm{kV}$. In order to deeply investigate these properties, we will combine these studies with very detailed analysis of the atomic configuration, spatial distribution and concentration of dopants via spatial-resolved EELS. Care was taken for avoiding/limiting damage of such nanostructures during the EELS and HR(S)TEM acquisitions.

This kind of studies has been possible due to the improvements developed the last two decades in transmission electron microscopes, allowing $\sim 100 \mathrm{meV}$ energy resolutions for a close to one angstrom electron beams and using low acceleration voltages. Thus, we have carried out these works using a FEI Titan - XFEG - Cs probe corrected microscope equipped with a monochromator (working at $80 \mathrm{KV}$ and with an energy resolution below $0.2 \mathrm{eV}$ ).

These N-doped nanotubes (CNx-NT) present different morphologies as a function of $\mathrm{N}$ concentration and configuration [2-4]. Thus, we have investigated the local dielectric properties considering these aspects that we have also analyzed in parallel. In this sense, Fig. 1 corresponds to the chemical and structural analysis of a multi-walled CNx-NT [8]. Furthermore, for properly analyzing the different spectroscopic modes, we have also taken into account other factors as the acquisition geometry (for probing the anisotropic effects), the thickness, the structural defects... Fig. 2 shows the low-loss EELS studies developed on this NT. The energy shifts of the different modes observed in this graphs are related to these aspects. All these results will be deeply discussed in the framework of previous experimental and theoretical works carried out in pure C-NT and compared with results coming from pure C-NT also collected under the same conditions [5-7]. In conclusion, the present study improves our knowledge of the dielectric/optoelectronic properties of $\mathrm{CNx}-\mathrm{NT}$ and provides further insight into the potential applications of these materials.

\section{References}

[1] "Boron-nitride and boron-carbonitride NTs: synthesis, characterization and theory", R. Arenal, X. Blase, A. Loiseau, Advances in Physics 59, 101 (2010).

[2] P. Ayala, R. Arenal, A. Rubio, A. Loiseau, T. Pichler, Rev. Mod. Phys. 82, 1843 (2010).

[3] P. Ayala, R. Arenal, M. Rummeli, A. Rubio, T. Pichler, Carbon 48, 575 (2010).

[4] C.P. Ewels, M. Glerup, J. Nanosci. Nanotech. 5, 1345 (2005). 
[5] D. Taverna et al., Phys. Rev. B 66, 235419 (2002).

[6] O. Stephan et al., Phys. Rev. B 66, 155422 (2002).

[7] R. Arenal, to be submitted (2013).

[8] CNx-MWNTs were produced via CVD by V.O. Khavrus (IFW, Dresden (Germ.)). We thank this team for having kindly provided these samples.
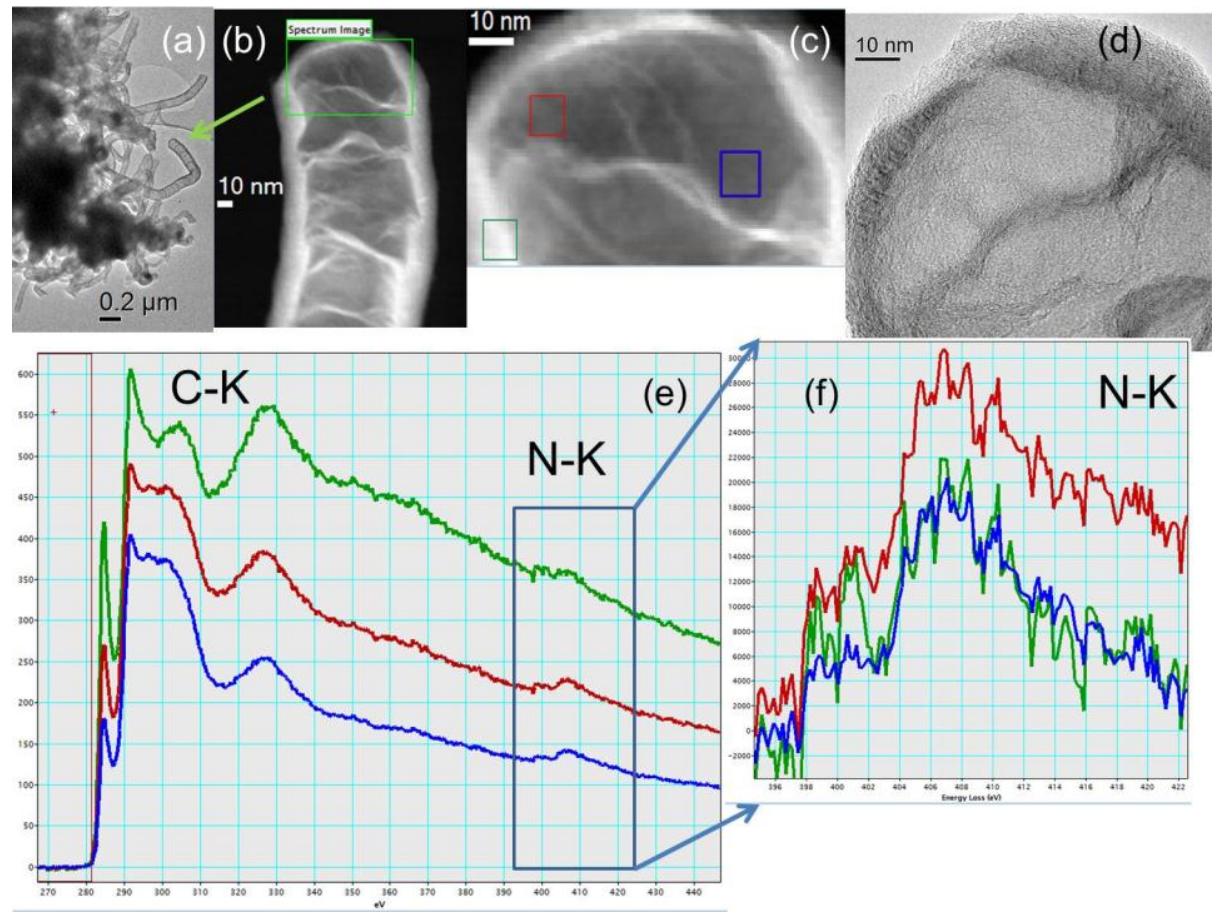

Figure 1. (a), Bright-field image of an agglomerate of CNx-MWNT. (b)-(d), HAADF and HRTEM images of an area (the tip) of one of these NT where spectroscopy data have been recorded (see Fig. 1 (b)). (e) EEL spectra extracted from the spectrum-image in the areas marked in Fig. 1 (c). C- and N-K edges are visible on these spectra. (f) Zoom of the N-K edge of these spectra, after background subtraction.

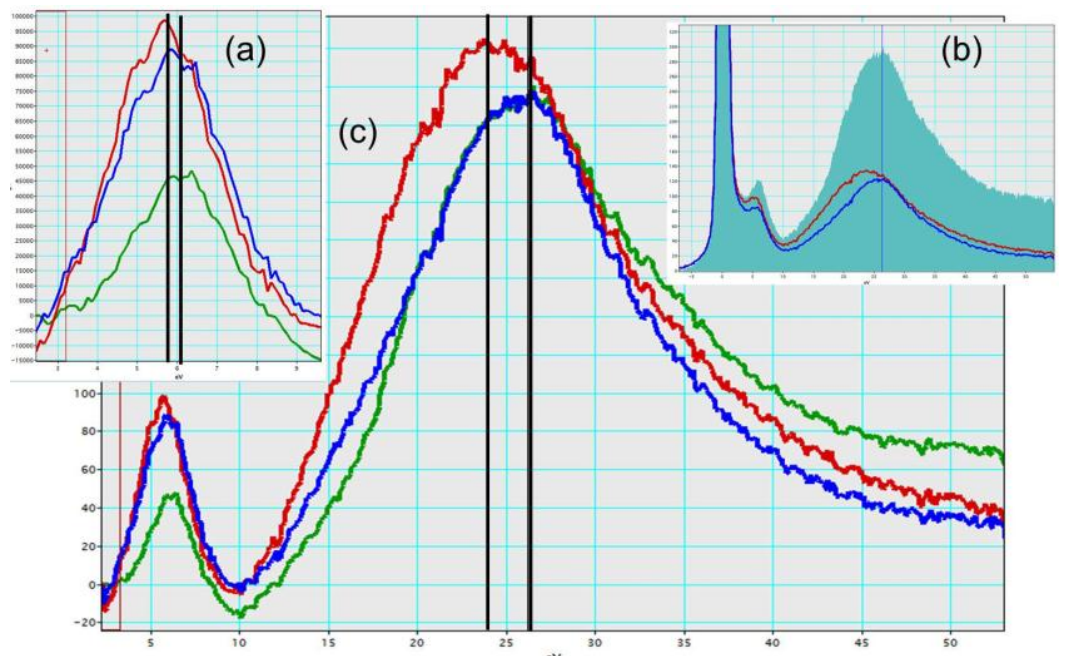

Figure 2. (a)-(c) Low-Loss EEL spectra extracted from a spectrum-image recorded in the same area of the previous one (Fig. 1). They have been extracted from the same areas that those marked in Fig. 1 (c), and they are: (b) the whole spectra after background subtraction, (b) a zoom in the spectral range of 2-10 eV, and (c) the raw spectra. 